\title{
MicroRNA-29b-3p promotes 5-fluorouracil resistance via suppressing TRAF5-mediated necroptosis in human colorectal cancer
}

\author{
Shuimei Wu, Yun Zhou, Ping Liu, Hui Zhang, Wanliang Wang, Yuan Fang, Xiang Shen \\ Department of Gastroenterology, Wuhu No.1 People's Hospital, Wuhu City, Anhui Province, China
}

\begin{abstract}
Drug resistance in colorectal cancer is a great challenge in clinic. Elucidating the deep mechanism underlying drug resistance will bring much benefit to diagnosis, therapy and prognosis in patients with colorectal cancer. In this study, miR-29b-3p was shown to be involved in resistance to 5-fluorouracil (5-FU)-induced necroptosis of colorectal cancer. Further, miR-29b-3p was shown to target a regulatory subunit of necroptosis TRAF5. Rescue of TRAF5 could reverse the effect of miR-29b-3p on 5-FU-induced necroptosis, which was consistent with the role of necrostatin-1 (a specific necroptosis inhibitor). Then it was demonstrated that miR-29b-3p was positively correlated with chemoresistance in colorectal cancer while TRAF5 negatively. In conclusion, it is deduced that miR-29b-3p/TRAF5 signaling axis plays critical role in drug resistance in chemotherapy for colorectal cancer patients by regulating necroptosis. The findings in this study provide us a new target for interfere therapy in colorectal cancer.
\end{abstract}

Key words: Colorectal cancer; miR-29b-3p; TRAF5; necroptosis; 5-fluorouracil resistance.

Correspondence: Dr. Xiang Shen, Department of Gastroenterology, Wuhu No.1 People's Hospital, Wuhu City, Anhui Province, China, Email: hao350318178@outlook.com

Contributions: SW, YZ contributed equally to this study. SW, YZ, XS, study concept; SW, YZ experiment completion; PL, HZ, WW, literature search; WW, YF, data analysis; PL, HZ, WW, YF, results discussion; SW, YZ, $\mathrm{XS}$, manuscript drafting and revision. All the authors have read and approved the final version of the manuscript and agreed to be accountable for all aspects of the work.

Conflict of interest: The authors declare that no conflicts of interest exist in this article.

Funding: This work was supported by the Wuhu Health commission Research Project

Ethics approval: All experiments were performed in the Wuhu No.1 People's Hospital and followed all guidelines. The Ethics Committee of the Wuhu No.1 People's Hospital formally approved this study.

Availability of data and material: All data generated or analyzed during this study are included in this published article. 


\section{Introduction}

Colorectal cancer (CRC) is a leading cause of cancer mortality and current strategies for treating this disease need to be improved. ${ }^{1-3}$ Fluorouracil, 5-fluorouracil (5-FU), is the most commonly used drug in clinic and is the backbone of all first-line therapies in adjuvant and metastatic therapy. ${ }^{4}$ Resistance to treatment is common, especially in metastatic settings, and understanding the mechanism that regulates 5-FU targets can help identify new therapeutic strategies to improve patient outcomes..$^{5-7}$

Cell susceptibility to apoptosis is severely impaired in cancer, while other forms of death become more important in cell killing and tumor responses to DNA-damaging factors. ${ }^{8}$ Necroptosis was a novel programmed form of necrotic cell death that bears a morphological resemblance to necrosis and a mechanistic resemblance to apoptosis. ${ }^{9}$ Receptor-interacting protein kinase 1 (RIP1) and RIP3-mixed lineage kinase domain (MLKD) -mediated necrosis are the most understood forms of regulated necroptosis..$^{10}$ Once the pro-apoptotic molecule fails to stimulate the apoptotic body, the default death program is triggered, inducing ripoptosome assembly leading to necrosis, which depends on the kinase activity of critical enzymes RIP1 and RIP3. ${ }^{11,12}$ Previous reports have shown that some compounds induce necroptotic cell death in multidrug-resistant cancer cell lines to ward off resistance, suggesting that necroptotic susceptibility is an inherent weakness of cancer. ${ }^{13-15}$ Therefore, screening for necroptosis inducers may be a novel strategy to treat 5-FU resistance in cancer.

It has been reported that alterations in microRNAs (miRNAs) contribute to the formation of tumor drug resistance, including 5-FU resistance. ${ }^{16-20}$ MiRNAs are small single-stranded non-coding RNA molecules coordinating complex pathological processes. ${ }^{18}$ These short non-coding RNAs act as post-transcriptional regulators of gene expression by binding to mRNAs containing
miRNA recognition elements. ${ }^{21}$ A single miRNA binding to its target mRNA can coordinate epigenetic regulation of gene products and promote developmental or pathological switches, such as cell survival and death. ${ }^{22-24}$ However, it is not clear how miRNAs are involved in mediating the formation of 5-FU resistance.

In this study, we investigated the role of miR-29b-3p in drugresistance of colorectal cancer to 5-FU in vitro and in vivo. Furthermore, the deep mechanism was explored by western blotting analysis.

\section{Materials and Methods}

\section{Cell culture}

Human colorectal cancer cell lines HT-29, HCT-116, SW480, SW620, LoVo, SW48, DLD-1, Caco-1 and HCT-15 cells were maintained in RMI-1640 (Gibco, Thermo Fisher Scientific, Waltham, MA, USA), supplemented with $10 \%$ FBS, $200 \mathrm{mM} \mathrm{L}-$ glutamine, $1 \% \mathrm{PS}$, and placed in a wet incubator at $37^{\circ} \mathrm{C}$ and $5 \%$ $\mathrm{CO}_{2}$. 5-FU (F6627-10G) was purchased from Sigma (Poole, UK), and soluble in dimethyl sulfoxide. Colorectal cancer cells were treated with $5-\mathrm{FU}$ at $5 \mu \mathrm{g} / \mathrm{mL}$.

\section{Western blot}

Western blotting is performed on the entire cell extract by lysis the cells in the RIPA buffer. Antibodies against TRAF5 (1:2000) and GAPDH (1:1500) were purchased from Abclonal (Shanghai, China). Primary antibodies were prepared in bovine serum albumin (BSA), tested with appropriate anti-mouse and anti-rabbit conjugates (Dako, Glostrup, Denmark; 1:5000) at room temperature for $1 \mathrm{~h}$, and visualized using the Enhanced Chemiluminescence Detection System (ECL) (Perkin Elmer, Waltham, MA, USA).
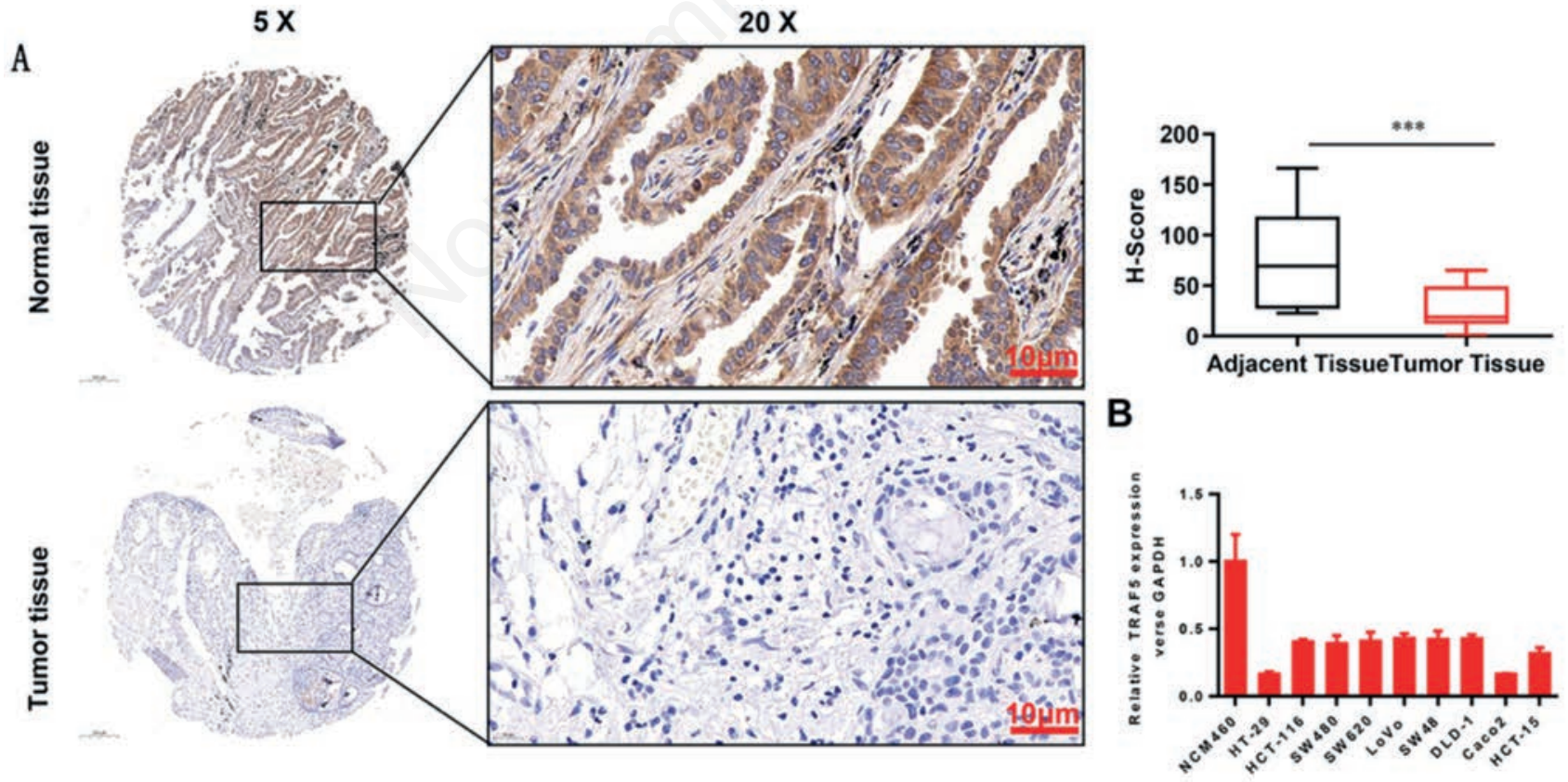

Figure 1. TRAF5 was significantly lower expressed in colon cancer tissues and cells. A) The protein level of TRAF5 in commercial colon tumor tissue microarray from 110 colon cancer samples was analyzed by immunohistochemistry. B) The expression level of TRAF5 mRNA in serials of colon cancer cell lines (HT-29, HCT-116, SW480, SW620, LoVo, SW48, DLD-1, Caco-1 and HCT-15) and a normal colorectal cell line (NCM480) were analyzed by real-time PCR. H-score means histochemistry score which indicates the intensity of positive staining. ${ }^{* * *} \mathrm{p}<0.001$ versus adjacent tissue. 


\section{Real-time PCR}

Total RNA was extracted by RNeasy Mini kit (Qiagen, Hilden, Germany) and first strain was synthesized by cDNA reverse transcription kit (Takara, Shiga, Japan). RT-PCR was performed by SYBRGreen method using ABI 7900 system (Applied Biosystems, Bedford, MA, USA). The protocol is as below: $95^{\circ} \mathrm{C}, 30 \mathrm{~s} ;\left(95^{\circ} \mathrm{C}\right.$, $15 \mathrm{~s} ; 60^{\circ} \mathrm{C}, 15 \mathrm{~s}$ ) for 40 cycles. The sequences of primers used are as follows: TRAF5, 5'-ACTCCGCGGATCCCAGATGATT3'(forward), 5'-CAGTTCCTTGTTCTGGGATCAC-3'(reverse) and GAPDH, 5'- TGACTTCAACAGCGACACCCA-3' (forward), 5'- CACCCTGTTGCTGTAGCCAAA-3' (reverse).

\section{Colony formation assay}

HCT-116 and LoVo cells were inoculated on 6-well plates at a density of $200 /$ well and cultured at $37^{\circ} \mathrm{C}$ for 10 days. Fixed with $4 \%$ paraformaldehyde for $10 \mathrm{~min}$ and stained with $0.1 \%$ crystal violet for $20 \mathrm{~min}$

\section{Flow cytometry}

An apoptosis detection kit (BD, 556547) was used to detect the percentage of apoptotic and necroptotic cells. Annexin V-FITC (excitation wavelength at $488 \mathrm{~nm}$, emission wavelength at $525 \mathrm{~nm}$ ) and PI (excitation wavelength at $488 \mathrm{~nm}$, emission wavelength at
$620 \mathrm{~nm}$ ) stained samples were measured by FACs (BD Biological Sciences, San Jose, CA, USA). The FITC-labeled cells were considered as apoptotic cells while PI-labeled cells were considered as necrotic cells. Necrosis inhibitor necrostatin-1 (NEC-1) was purchased from Selleck (CAT: 852391-15-2, Shanghai, China) and was used to treat colorectal cancer cells at $600 \mathrm{nmol} / \mathrm{L}$.

\section{Immunohistochemical analysis}

Colon tumor tissue microarray was purchased from OUTDO Biotech Co. Ltd. (catalog: HCoIA120cs01, Shanghai, China). The microarray was deparaffinized, subjected to antigen retrieval in sodium citrate buffer with $1 \mathrm{M}$ citric acid, boiling for $20 \mathrm{~min}$ at $97^{\circ} \mathrm{C}$, incubated with methanol and $30 \%$ hydrogen peroxide for 30 min at room temperature followed by BSA blockade for $35 \mathrm{~min}$ at room temperature. Then the microarray was incubated with primary antibody against TRAF5 (dilution 1:50, R\&D systems, USA) at $4^{\circ} \mathrm{C}$ overnight, placed for $1 \mathrm{~h}$ at room temperature with rabbit EnVisionamplification reagent (K4003, Dako), incubated with diaminobenzidine solution for 5 min at room temperature, counterstained for 8 min with Hematoxylin (Dako) at room temperature, and dehydrated in graded ethanol washes $(70,85,95,100 \%)$ for 1 $\min$ followed by $5 \mathrm{~min}$ in xylene.
A

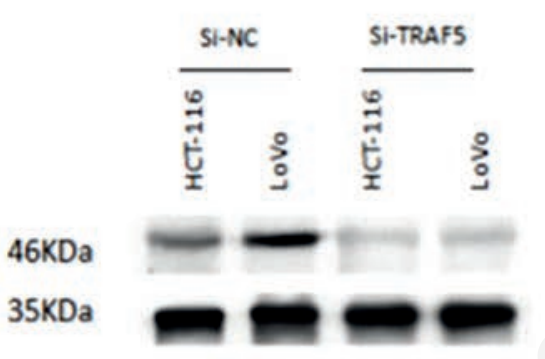

C

GAPDH

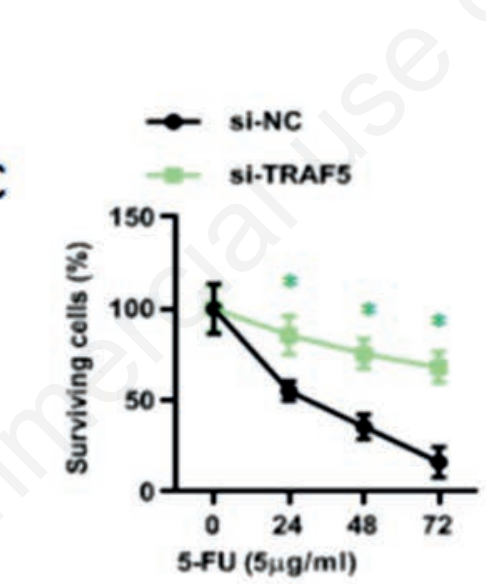

D

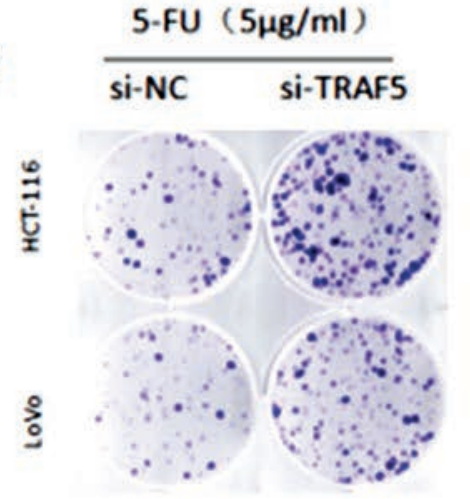

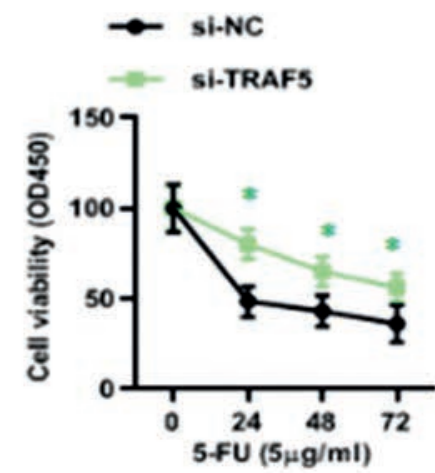

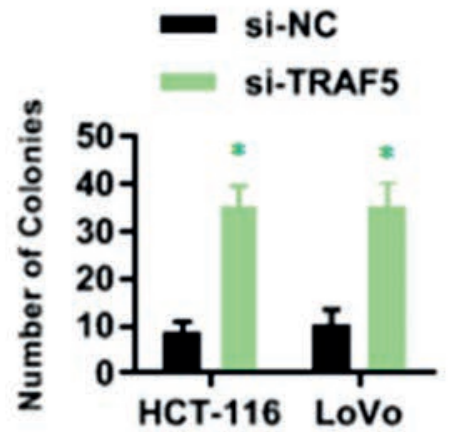

Figure 2. TRAF5 contributed to the 5-FU resistance in colon cancer cells. A) The efficiency of TRAF5 knock-down in HCT-116 and LoVo cells were analyzed by real-time PCR. B) The efficiency of TRAF5 knock-down in HCT-116 and LoVo cells were verified by Western blot. C) The effect of TRAF5 knock-down on the cell viability of HCT-116 and LoVo cells were analyzed by CCK-8. D) The effect of TRAF5 knock-down on the colony formation of HCT-116 and LoVo cells were analyzed by colony formation assay. ${ }^{*}$ p $<0.05$ versus si-NC group. 


\section{Construction of plasmid and lentivirus pakage}

MiR-29b-3p mimics were synthesized by Genepharm (Shanghai, China). Plasmid carrying 3-UTR of TRAF5 was chemical synthesized by Generay Company (Shanghai, China). Lentivirus carrying target gene was produced in 293T cells with pspax2 and pMD2.G as helper plasmid.

\section{Luciferase reporter assay}

Cancer cells were treated with either TRAF5 wild type (WT) or mutated-type (Mut) promoter reporters in combination with miR-29b-3p mimic. $48 \mathrm{~h}$ after transfection, the luciferase activity was detected by the Dual Luciferase Reporter Assay System (Promega, Madison, WI, USA).

\section{RNA pull-down assay}

Pierce ${ }^{\mathrm{TM}}$ Magnetic RNA-Protein Pull-Down Kit (\#20164, ThermoFisher Scientific) was used for RNA Immunoprecipitatioin (RIP) assay. In brief, RNA was bound to the beads to receive the RNA for protein binding. Then, the RNA-bound beads were balanced in Protein-RNA Binding Buffer before the addition of the protein lysate. The beads were then washed and separated after vortex on a magnetic stand. Lastly, RT-qPCR assay was used to assess the specificity of RNAs pulled.

\section{Xenograft models}

Male BALB/c NOD mice aged 4 to 6 weeks were purchased from Shanghai Experimental Animal Center of China. CRC cells $\left(3 \times 10^{5}\right.$ cells, $200 \mu \mathrm{L}$ normal saline) were injected into mice; 5 -FU at dose of $10 \mathrm{mg} / \mathrm{kg}$ or normal saline was intraperitoneally injected after 10 days, before starting 5-FU therapy, intratumoral injections of miR-29b-3p inhibitors or si-TRAF5 were administered. They were tested every 5 days and killed 4 weeks after administration. Tumor volume was measured with digital calipers and tumor volume was calculated: volume $=0.5 \times$ width $^{2} \times$ length .

\section{Statistical analysis}

Data are presented as Mean $\pm \mathrm{SD}$. Statistical analysis was performed using GraphPad Prism 9 software (La Jolla, CA, USA). The difference in the mean between the groups was assessed using either the Student's $t$-test or one-way analysis of variance; $p<0.05$ was considered statistically significant.
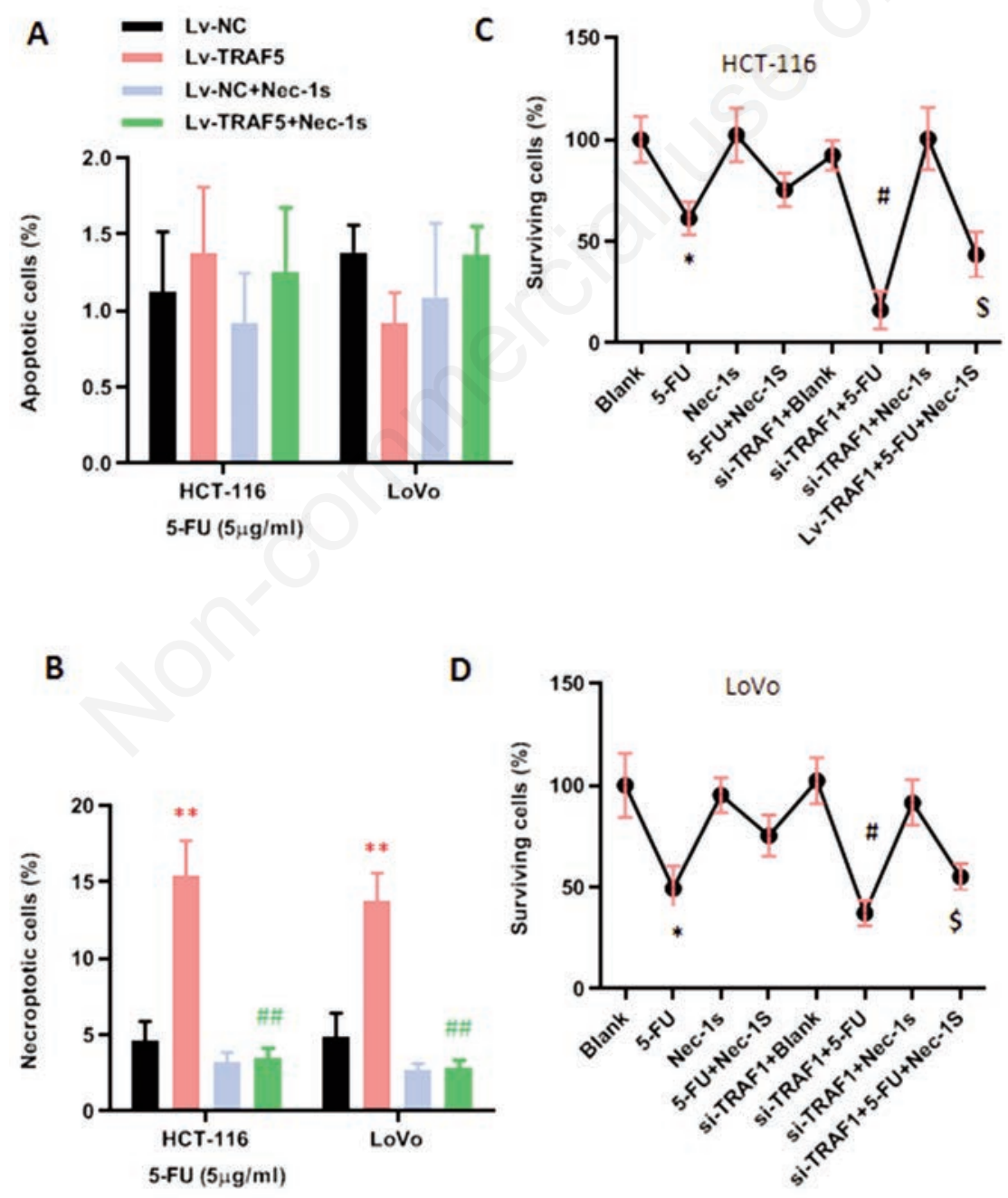

Figure 3. TRAF5 improves the 5-FU induced necroptosis in colon cancer cells. A) The apoptotic cell rate of HCT-116 and LoVo cells were analyzed by FACs; B. Thenecroptotic cell rate of HCT-116 and LoVo cells were analyzed by FACs; C. The cell survival rate of HCT116 cells were analyzed by CCK-8 assay. D. The cell survival rate of LoVo cells were analyzed by CCK-8 assay. The FITC-labeled cells were considered as apoptotic cells while PI-labeled cells were considered as necrotic cells. ${ }^{*} \mathrm{p}<0.05,{ }^{* *} \mathrm{p}<0.01$ versus $\mathrm{Blank}$ group; $\# \mathrm{pP}<\mathbf{0 . 0 5}$, \#\# $\mathrm{p}<\mathbf{0 . 0 5}$ versus 5-FU group; $\mathbf{P}<\mathbf{0 . 0 5}$ versus si-TRAF+5-FU group. 


\section{Results}

TRAF5 was significantly lower expressed in colon cancer tissues and cells

To investigate the expression of TRAF5, a key regulator of necrosis in colon cancer, we performed immunohistochemistry in commercial colon tumor tissue microarray from 110 colon cancer samples (Figure 1A). As shown, we observed positive staining of TRAF5 $(>90 \%)$ in the cytoplasm of adjacent tissues, suggesting that TRAF5 is normally expressed in normal human colon tissue, whereas it was negative in colon cancer tissues. We then evaluated the expression level of TRAF5 in colon cancer cell lines. As shown in Figure 1B, the expression of TRAF5 in colon cancer cell lines including HT-29, HCT-116, SW480, SW620, LoVo, SW48, DLD1, Caco-1 and HCT-15, was lower than normal colorectal cells (NCM480). These results demonstrate that TRAF5 was much lower expressed in colon cancer tissues and cells.

TRAF5 contributed to the 5-FU resistance in colon cancer cells

To evaluate the role of TRAF5 in the sensitivity of colon cancer cells to 5-FU, we knocked down the expression of TRAF5 with transfection of specific siRNAs into HCT-116 cells and LoVo cells. The silence efficiency was confirmed by real-time PCR (Figure 2A) and Western Blot (Figure 2B). Notably, the silence of TRAF5 remarkably increased the survival rate (Figure $2 \mathrm{C}$ ) and formatted colonies (Figure 2D) in 5-FU-treated HCT-116 cells and LoVo cells. Thus, these results demonstrated that TRAF5 contributed to the 5-FU resistance in HCT-116 cells and LoVo cells.
TRAF5 improved the 5-FU induced necroptosis in colon cancer cells

To investigate whether necrosis was associated with impaired TRAF5-5-FU resistance, TRAF5 was overexpressed in HCT-116 cells and LoVo cells (Figure S1A). As shown, overexpression of TRAF5 did not affect the number of apoptotic cells in 5-FU-treated HCT-116 cells and LoVo cells (Figure 3A), but significantly increased the number of necroptotic cells in 5-FU-treated HCT-116 cells and LoVo cells (Figure 3B). Furthermore, this activated necrosis was eliminated by the necrosis inhibitor necrostatin-1 (NEC1). Similarly, after 5-FU treatment, TRAF5 overexpression significantly reduced the survival rate of HCT-116 (Figure 3C) and LoVo (Figure 3D) cells, while NEC-1 reversed this trend. These results suggest that TRAF5-mediated necrosis is inhibited in 5-FU resistant HCT-116 cells and LoVo cells.

miR-29b-3p was high expressed in colon cancer and suppressed the expression of TRAF5 via targeting its 3'UTR

To identify the potential regulatory miRNAs of TRAF5 in colon cancer, RNA immunoprecipitation (RIP) assay was performed and found a miR-29b-3p enrichment in the component of TRAF5 3'UTR (Figure 4A). In order to prove that TRAF5 is a direct target gene of miR-29b-3p, we constructed a fluorescent reporter gene vector at the miR-29b-3p binding site on TRAF53'UTR. We examined luciferase activity by transfection of miR29b-3p with TRAF5-3'UTR reporter vector into colon cancer cells. At the same time, we performed point mutation on TRAF53'UTR to construct mutant TRAF5-3'UTR fluorescent reporter plasmid. As shown, miR-29b-3p was increased significantly (Figure S1B) and dramatically decreased fluorescence activity,
A

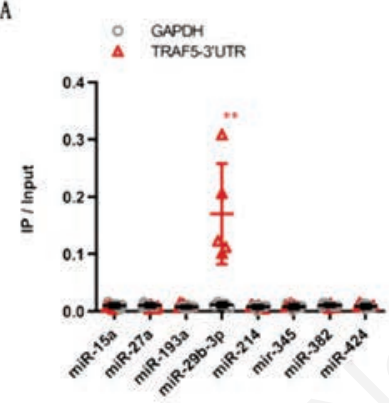

D

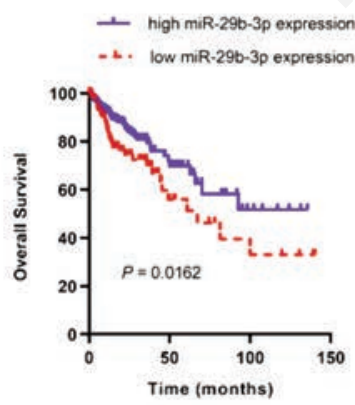

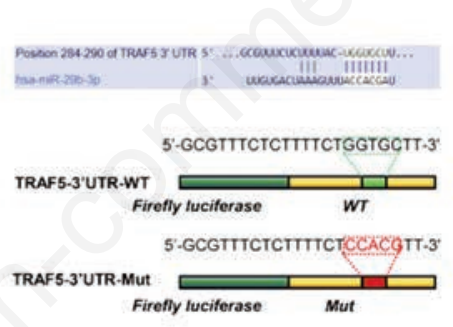

E

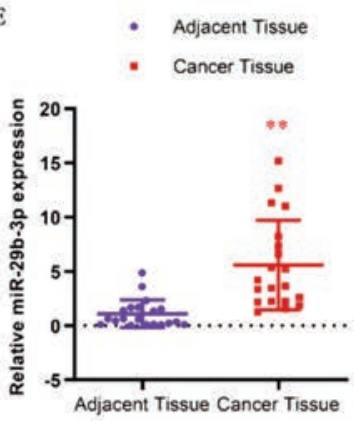

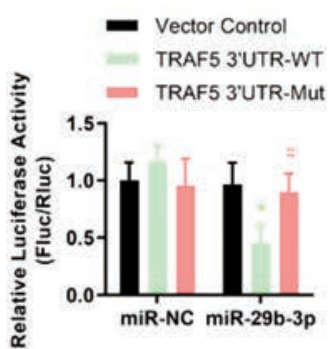

C
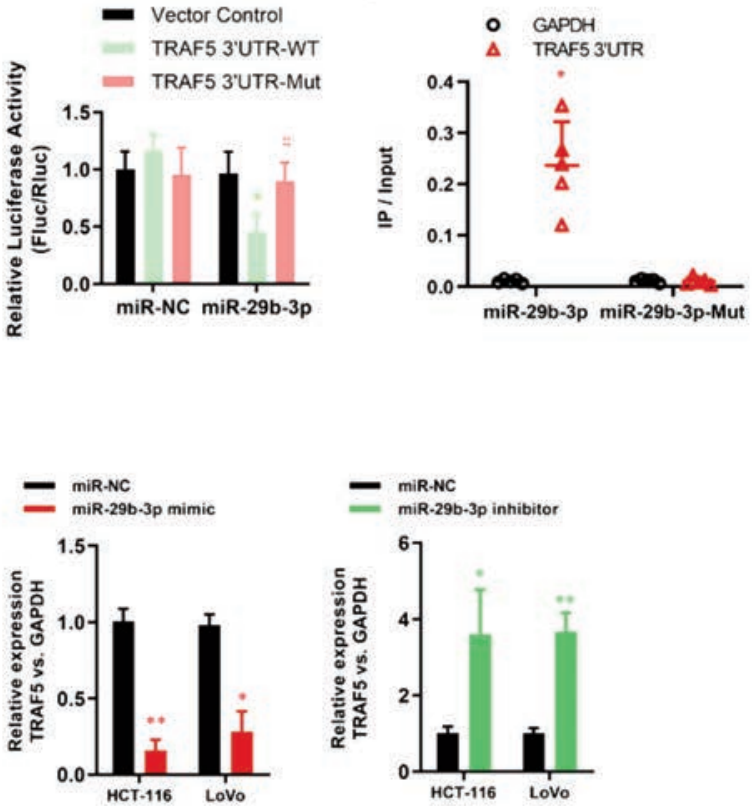

Figure 4. miR-29b-3p is high expressed in colon cancer and suppressed the expression of TRAF5 via targeting its 3 'UTR. A) The TRAF5 3'UTR enriched miRNAs was screened by RNA IP. B) Dual-luciferase assay was used to evaluate the targeting of miR-29b-3p to TRAF5 3'UTR. C) Wild-type and mutant miR-29b-3p RNA pull down was used to verify the interacting between miR-29b-3p and TRAF5 3'UTR. D) The level of miR-29b-3p in colon cancer tissues was analyzed by real-time PCR. E) Kaplan-Meier analyses was used to evaluate the correlation between miR-29b-3p expression and the overall survival in the colon cancer patients. F) The effects of miR-29b$3 \mathrm{p}$ over-expression or silence on the level of TRAF5 were evaluated by real-time $\mathbf{P C R} .{ }^{*} \mathbf{p}<0.05,{ }^{* *} \mathrm{p}<0.01$ versus control group; \# $\mathbf{p}<0.05$ versus TRAF5-3'UTR-WT group. 
while there was no significant change in fluorescence activity in mutant UTR (Figure 4B). Moreover, the wild-type and mutant miR-29b-3p RNA Pull Down confirmed that TRAF5-3'UTR was enriched in the wild-type miR-29b-3p component (Figure 4C). Moreover, we found that miR-29b-3p are highly expressed in the colon cancer tissues (Figure 4D) and the up-regulated expression of which is related to the poor prognosis of patients with colon cancer (Figure 4E). Furthermore, miR-29b-3p mimics reduced the expression of TRAF5 in HCT-116 cells and LoVo cells, whereas miR-29b-3p inhibitor increased its level (Figure 4F). These results demonstrate that miR-29b-3p is high expressed in colon cancer and suppressed the expression of TRAF5 via targeting its 3'UTR.

miR-29b-3p inhibited the 5-FU induced necroptosis and improved5-FU resistance in colon cancer cells via suppressing TRAF5

To determine whether TRAF5-mediated necrosis was related to 5 -FU resistance triggered by miR-29b-3p, we reduced the expression of miR-29b-3p and TRAF5. As seen in Figure 5A, inhibition of miR-29b-3p significantly increased the number of necroptotic cells in HCT-116 and LoVo cells, while silencing of TRAF5 completely reversed this situation.

In order to investigate the role of miR-29b-3p damaged necro- sis in the development of 5-FU resistance of HCT-116 cells and LoVo cells in vivo, HCT-116 cells inhibited or not inhibited by miR-29b-3p were subcutaneously injected into nude mice and then treated with the optimal dose of 5-FU. We found that inhibition of miR-29b-3p significantly improved the chemotherapy effect of 5FU, while silencing of TRAF5 had little effect on tumor growth (Figure 5B). The final tumor size in the miR-29b-3p inhibited group was significantly smaller than that in the 5-FU alone group, and the silencing of TRAF5 further reversed this result (Figure $5 B$ ). In addition, average tumor weight was significantly heavier in the TRAF5-silenced group than the 5-FU group alone (Figure 5B). In summary, these results suggested that miR-29b-3p inhibited 5FU-induced necrosis by inhibiting TRAF5 in vivo and in vitro, and increased the resistance of colon cancer cells to 5-FU.

\section{Discussion}

miR-29b-3p is abnormally expressed in a variety of cancers. ${ }^{25,} 26$ For example, in breast cancer, miR-29b-3p promotes the occurrence and development of triple negative breast cancer by inhibiting the expression of TRAF3. ${ }^{27}$ Studies have shown that the
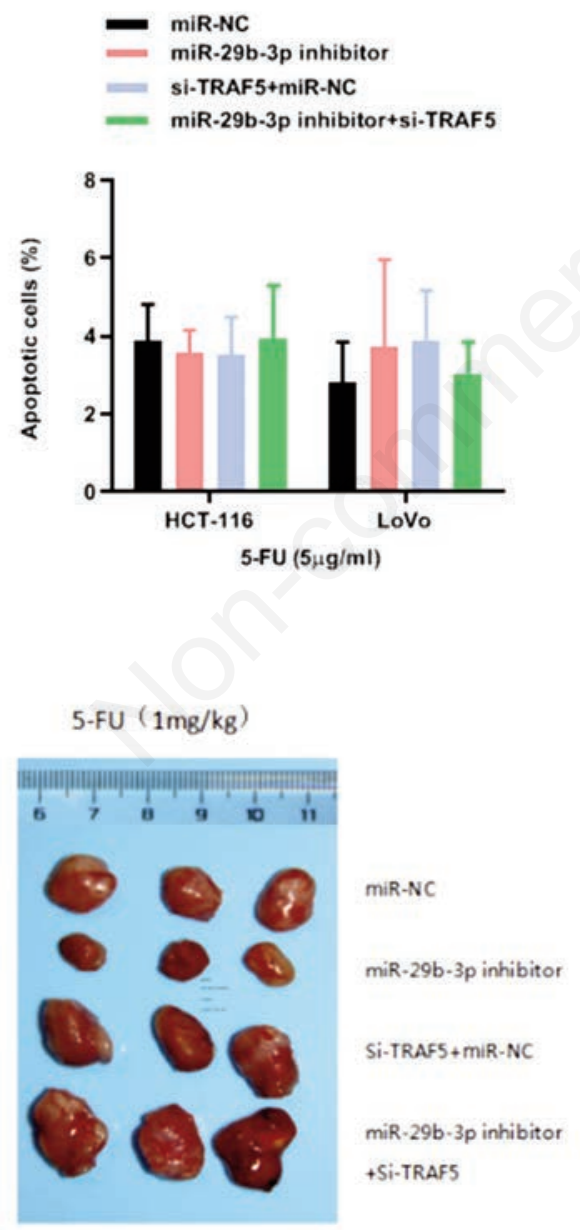
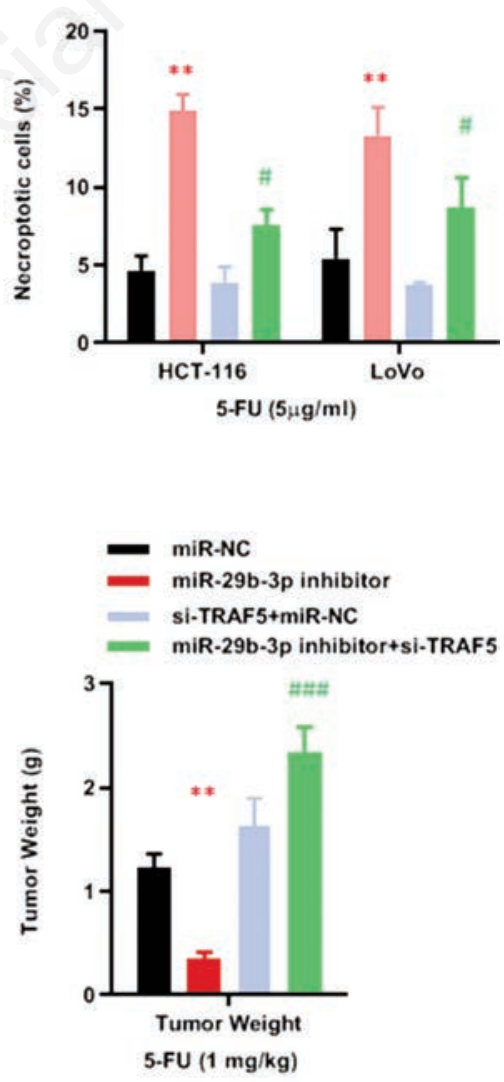

Figure 5. miR-29b-3p inhibits the 5-FU induced necroptosis and improves 5-FUresistance in colon cancer cells via suppressing TRAF5 in vitro and in vivo. A) The apoptotic and necroptotic cell rate of HCT-116 and LoVo cells were analyzed by FACs. B) The representative tumor image and tumor weight generated from the TRAF5 silence with or without miR-29b-3p knock-down HCT-116 cells were shown. The FITC-labeled cells were considered as apoptotic cells while PI-labeled cells were considered as necrotic cells. ${ }^{* *} \mathrm{p}<0.01$ versus miR-NC group; \# $\mathrm{p}<0.05$ versus $\mathrm{miR}-\mathrm{NC}$ group; \#\# $\mathrm{p}<0.001$ versus $\mathrm{miR}-29 \mathrm{~b}-3 \mathrm{p}$ inhibitor group. 
expression of miR-29b-3p is increased in human colon cancer, which activates EMT and promotes the proliferation and invasion of colon cancer cells. ${ }^{28}$ This is consistent with our results. We found that the level of miR-29b-3p was related to the sensitivity of cells to 5-FU. Inhibition of miR-29b-3p can enhance cell resistance to 5-FU. In this study, we proved for the first time that miR-29b$3 p$ contributed to the progression of colorectal cancer and inhibition of miR-29b-3p may be a new way to treat colorectal cancer.

Tumor necrosis factor receptor-related factors (TRAFs), including TRAF1, 2, 3, 5, and 6, have been identified as molecular activator of NF-kB. TRAFs act as ubiquitin ligases through induction K-63 poly-ubiquitination in receptor-interacting protein 1 (RIP1), which leads to activation of the transforming growth factor B-activated kinase-1 (TAK1)/ table 2/3 complex. Activated TAK1/TAB2/3 phosphorylates and activates the NF-kB kinase inhibitor (IKK)-a/b/g complex, leading to nuclear translocation and activation of NF-kB. ${ }^{29}$ A growing body of evidence suggests that TRAF5 plays a key role in cancer progression. ${ }^{26,30-32}$ For example, the expression of TRAF5 in gastric cancer tissues has been shown to be up-regulated, and its high expression predicts poor overall survival. ${ }^{33}$ Over-expression of TRAF5 promotes in vitro migration of gastric cancer cells. Down-regulation of TRAF5 after over-expression of miR-26b can significantly inhibit the proliferation of melanoma cells and promote apoptosis. ${ }^{33}$ Conversely, we found that TRAF5 expression was lower in CRC tissues than in adjacent tissue. Moreover, we found that miR-29-3p was resistant to 5-FU induced necroptosis in vitro and reduced the ability of 5FU to inhibit tumor growth in xenograft mouse models in vivo. Further studies showed that miR-29-3p regulated drug resistance by down-regulating the expression of TRAF5, the regulatory subunit of necroptosis. However, in miR-29-3p expressed cells, the expression of TRAF5 can re-sensitize colon cancer cells to 5-FUinduced necrosis (Figure 6). These findings suggest that inhibition of miR-29-3p or restoration of TRAF5 expression may have significant therapeutic potential for overcoming drug resistance in patients with colorectal cancer. However, more experiments are necessary to further support the role of miR-29-3p in colorectal cancer. For example, the downstream signaling of miR-29$3 \mathrm{p} /$ TRAF5 is not clear, but it will be very important to elucidate the deep molecular mechanism. In addition, the clinical role of miR29-3p and/or TRAF5 in therapy or diagnosis of colorectal cancer is also not known. All these issues are our focus point in future work.

\section{Acknowledgements}

Thanks for the language edit by Dr. Roshan Shrestha from Department of Genetics in National Cancer Institute, USA.
5-FU Resistance

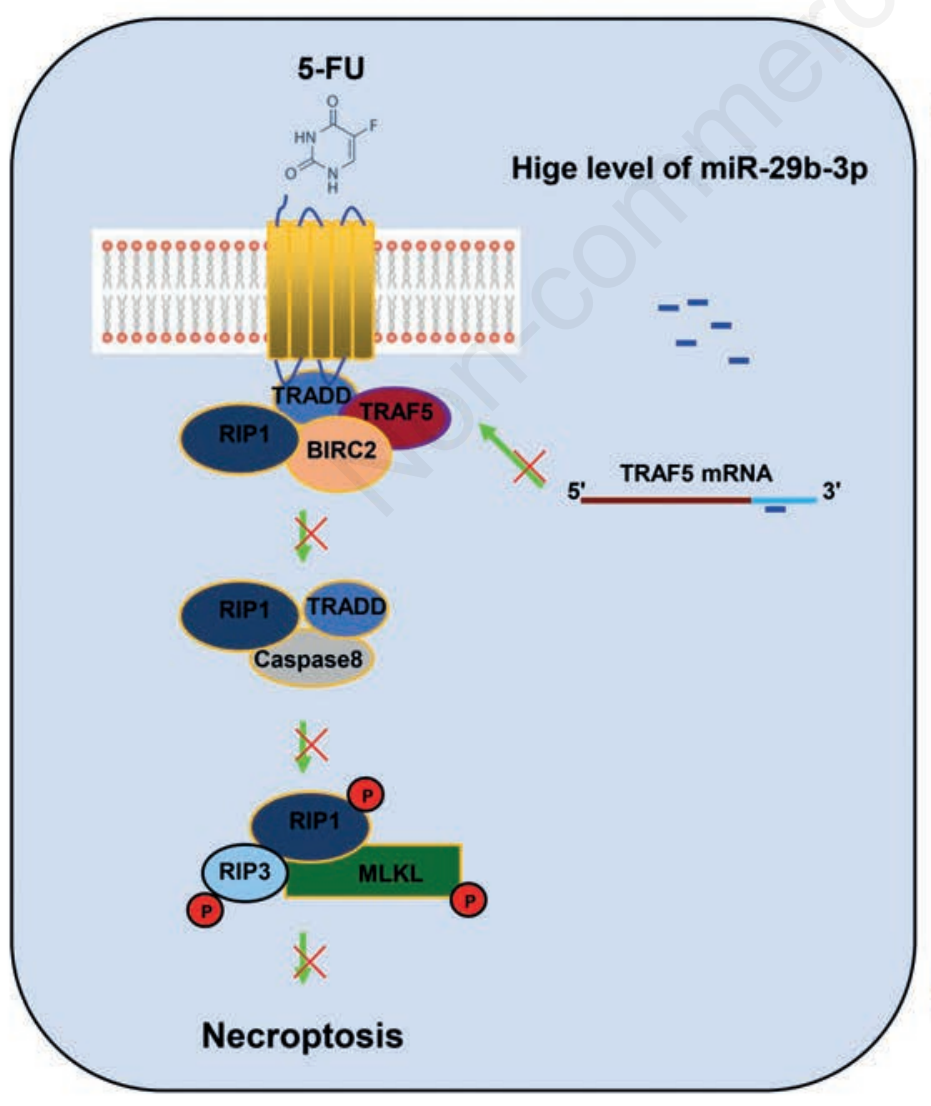

5-FU Sensitive

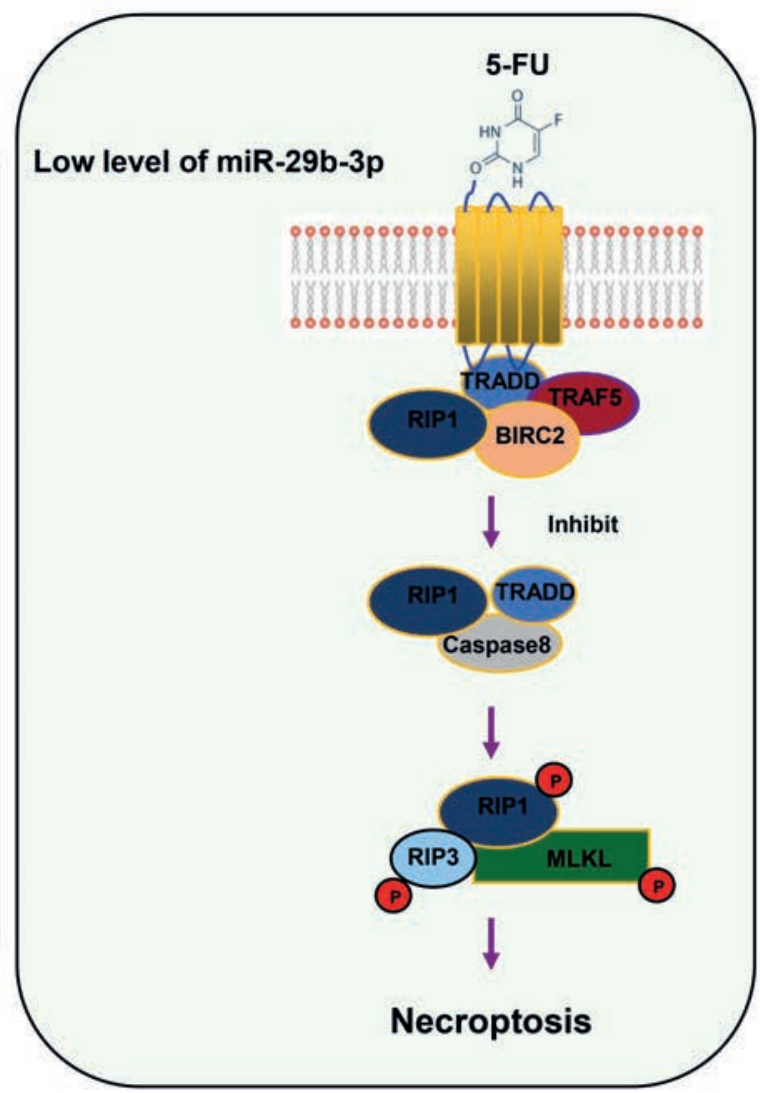

Figure 6. A schematic model showed the important role of miR-29-3p/TRAF5/necroptosis signaling axis in colorectal cancer. 


\section{References}

1. Janney A, Powrie F, Mann EH. Host-microbiota maladaptation in colorectal cancer. Nature 2020;585:509-17.

2. Rehman SK, Haynes J, Collignon E, Brown KR, Wang Y, Nixon AML, et al. Colorectal cancer cells enter a diapause-like DTP state to survive chemotherapy. Cell 2021;184: 226-42 e221.

3. Siegel RL, Miller KD, Fuchs HE, Jemal A. Cancer statistics, 2021. CA Cancer J Clin 2021;71:7-33.

4. Longley DB, Harkin DP, Johnston PG. 5-fluorouracil: mechanisms of action and clinical strategies. Nat Rev Cancer 2003;3:330-8.

5. Adlard JW, Richman SD, Seymour MT, Quirke P. Prediction of the response of colorectal cancer to systemic therapy. Lancet Oncol 2002;3:75-82.

6. Filipits M, Pohl G, Rudas M, Dietze O, Lax S, Grill R, et al. Clinical role of multidrug resistance protein 1 expression in chemotherapy resistance in early-stage breast cancer: the Austrian Breast and Colorectal Cancer Study Group. J Clin Oncol 2005;23:1161-8.

7. Leichman CG, Lenz HJ, Leichman L, Danenberg K, Baranda J, Groshen S, et al. Quantitation of intratumoral thymidylate synthase expression predicts for disseminated colorectal cancer response and resistance to protracted-infusion fluorouracil and weekly leucovorin. J Clin Oncol 1997;15:3223-9.

8. Tummers B, Mari L, Guy CS, Heckmann BL, Rodriguez DA, Ruhl S, et al. Caspase-8-dependent inflammatory responses are controlled by its adaptor, FADD, and necroptosis. Immunity 2020; ;52(6):994-1006.e8.

9. Gong YT, Fan ZY, Luo GP, Yang C, Huang QY, Fan K, et al. The role of necroptosis in cancer biology and therapy. Mol Cancer 2019;18:100.

10. Cremolini C, Rossini D, Dell'Aquila E, Lonardi S, Conca E, Del Re M, et al. Rechallenge for patients with RAS and BRAF wild-type metastatic colorectal cancer with acquired resistance to first-line cetuximab and irinotecan: A phase 2 single-arm clinical trial. JAMA Oncol 2019;5:343-50.

11. Fresquet V, Garcia-Barchino MJ, Larrayoz MJ, Celay J, Vicente C, Fernandez-Galilea M, et al. Endogenous retroelement activation by epigenetic therapy reverses the Warburg effect and elicits mitochondrial-mediated cancer cell death. Cancer Discov 2020;11:1268-85.

12. Zhou B, Zhang JY, Liu XS, Chen HZ, Ai YL, Cheng K, et al. Tom20 senses iron-activated ROS signaling to promote melanoma cell pyroptosis. Cell Res 2018;28:1171-85.

13. Newton K, Wickliffe KE, Maltzman A, Dugger DL, Reja R, Zhang Y, et al. Activity of caspase- 8 determines plasticity between cell death pathways. Nature 2019;575:679-82.

14. Paludan SR, Reinert LS, Hornung V. DNA-stimulated cell death: implications for host defence, inflammatory diseases and cancer. Nat Rev Immunol 2019;19:141-53.

15. Seehawer M, Heinzmann F, D'Artista L, Harbig J, Roux PF, Hoenicke L, et al. Necroptosis microenvironment directs lineage commitment in liver cancer. Nature 2018;562:69-75.
16. Mahawongkajit P, Tomtitchong P. Expression of miRNA in 5FU resistant esophageal cancer. Mol Clin Oncol 2020;13:221-7.

17. Meng X, Fu R. miR-206 regulates 5-FU resistance by targeting Bcl-2 in colon cancer cells. Onco Targets Ther 2018;11:1757-65.

18. Wang CQ. MiR-195 reverses 5-FU resistance through targeting HMGA1 in gastric cancer cells. Eur Rev Med Pharmacol Sci 2019;23:3771-8.

19. Xu F, Ye ML, Zhang YP, Li WJ, Li MT, Wang HZ, et al. MicroRNA-375-3p enhances chemosensitivity to 5-fluorouracil by targeting thymidylate synthase in colorectal cancer. Cancer Sci 2020;111:1528-41.

20. Yang Y, Bao Y, Yang GK, Wan J, Du LJ, Ma ZH. MiR-214 sensitizes human colon cancer cells to 5-FU by targeting Hsp27. Cell Mol Biol Lett 2019;24:22.

21. Fyfe I. MicroRNAs - diagnostic markers in Parkinson disease? Nat Rev Neurol 2020;16:65.

22. Ji C, Guo X. The clinical potential of circulating microRNAs in obesity. Nat Rev Endocrinol 2019;15:731-43.

23. Jung G, Hernandez-Illan E, Moreira L, Balaguer F, Goel A. Epigenetics of colorectal cancer: biomarker and therapeutic potential. Nat Rev Gastroenterol Hepatol 2020;17:111-30.

24. Stower H. An miRNA linked to metabolic disease. Nat Med 2020;26:1677.

25. Jia R, Wang C. MiR-29b-3p reverses cisplatin resistance by targeting COL1A1 in non-small-cell lung cancer A549/DDP cells. Cancer Manag Res 2020;12:2559-66.

26. Zhao C, Li Y, Hu X, Wang R, He W, Wang L, et al. LncRNA $\mathrm{HCP} 5$ promotes cell invasion and migration by sponging miR$29 \mathrm{~b}-3 \mathrm{p}$ in human bladder cancer. Onco Targets Ther 2020;13:11827-38.

27. Zhang B, Shetti D, Fan C, Wei K. miR-29b-3p promotes progression of MDA-MB-231 triple-negative breast cancer cells through downregulating TRAF3. Biol Res 2019;52:38.

28. Ding D, Li C, Zhao T, Li D, Yang L, Zhang B. LncRNA H19/miR-29b-3p/PGRN axis promoted epithelial-mesenchymal transition of colorectal cancer cells by acting on wnt signaling. Mol Cells 2018;41:423-35.

29. Tao T, Cheng C, Ji Y, Xu G, Zhang J, Zhang L, et al. Numbl inhibits glioma cell migration and invasion by suppressing TRAF5-mediated NF-kappaB activation. Mol Biol Cell 2012;23:2635-44.

30. Gong H, Fang L, Li Y, Du J, Zhou B, Wang X, et al. miR873 inhibits colorectal cancer cell proliferation by targeting TRAF5 and TAB1. Oncol Rep 2018;39:1090-8.

31. Horie R, Watanabe T, Ito K, Morisita Y, Watanabe M, Ishida T, et al. Cytoplasmic aggregation of TRAF2 and TRAF5 proteins in the Hodgkin-Reed-Sternberg cells. Am J Pathol 2002;160:1647-54.

32. Liang Z, Li X, Liu S, Li C, Wang X, Xing J. MiR-141-3p inhibits cell proliferation, migration and invasion by targeting TRAF5 in colorectal cancer. Biochem Biophys Res Commun 2019;514:699-705.

33. Chen Z, Zhao L, Zhao F, Yang G, Wang J. MicroRNA-26b regulates cancer proliferation migration and cell cycle transition by suppressing TRAF5 in esophageal squamous cell carcinoma. Am J Transl Res 2016;8:1957-70.

Received for publication: 16 March 2021. Accepted for publication: 21 May 2021.

This work is licensed under a Creative Commons Attribution-NonCommercial 4.0 International License (CC BY-NC 4.0).

(C) Copyright: the Author(s), 2021

Licensee PAGEPress, Italy

European Journal of Histochemistry 2021; 65:3247

doi:10.4081/ejh.2021.3247

[European Journal of Histochemistry 2021; 65:3247] 